\title{
Biomechanical study on the stress distribution of the knee joint after distal femoral fracture malunion with residual varus-valgus deformity
}

\section{Ming Li}

the Third Hospital of Hebei Medical University

Pan $\mathrm{Hu}$

Tongzhou Campus of Peking University People's Hospital

\section{Lijie Ma}

the Third Hospital of Hebei Medical University

\section{Di Zhang}

the Third Hospital of Hebei Medical University

\section{Wenli Chang}

Cangzhou Hospital of Integrated TCM-WM Hebei

\section{Yanbin Zhu}

the Third Hospital of Hebei Medical University

\section{Tao Zhang}

Guizhou Orthopedics Hospital

\section{Ning Wei}

the Fourth Hospital of Shijiazhuang

Wei Chen ( $\nabla$ drchenwei1@163.com)

Hebei Medical University Third Affiliated Hospital https://orcid.org/0000-0001-6455-0755

\section{Research article}

Keywords: Femoral fracture, Malunion, Varus deformity, Valgus deformity, Biomechanics, Knee joint

Posted Date: May 7th, 2020

DOl: https://doi.org/10.21203/rs.3.rs-24051/v1

License: (c) (i) This work is licensed under a Creative Commons Attribution 4.0 International License. Read Full License 


\section{Abstract \\ Background}

To investigate the effect of residual varus and valgus deformity on the stress distribution of knee joint after distal femoral fracture malunion.

\section{Methods}

Fourteen adult cadaver specimens with formalin were selected to establish the femoral fractures models, which were fixed subsequently at neutral position (anatomical reduction) and malunion positions (at 3 degrees, 7 degrees, 10 degrees valgus positions and 3 degrees, 7 degrees, and 10 degrees varus positions). The stress distribution on the medial and lateral plateau of the femur was quantitatively measured using ultra-low pressure sensitive film technology. The change of stress distribution of knee joint after femoral fracture malunion and the relationship between stress value and residual varus varus or valgus deformity were analyzed.

\section{Results}

Under $400 \mathrm{~N}$ vertical load, the stress values on the medial and lateral plateau of the femur at the neutral position were $1.162 \pm 0.114 \mathrm{MPa}$ and $1.103 \pm 0.144 \mathrm{MPa}$, respectively. When compared with the stress values measured at the neutral position, the stress on the medial plateau of femur were significantly higher at varus deformities and lower at valgus deformities, and the stress on the lateral plateau was significantly higher at valgus deformity and lower at varus deformities (all $P<0.05$ ). The stress values on the medial plateau of tibia were significantly higher than the corresponding data on the lateral plateau at neutral and 3 degrees, 7 degrees, 10 degrees varus deformities, respectively (all $P<0.05$ ), and significantly lower than the corresponding data on the lateral plateau at 3 degrees, 7 degrees, 10 degrees valgus deformities, respectively (all $P<0.05$ ).

\section{Conclusions}

Residual varus and valgus deformity after femoral fracture malunion can cause obvious changes of the stress distribution of knee joint. Therefore, the distal femoral fracture should be anatomically reduced and rigidly fixed to avoid residual varus-valgus deformity and malalignment of lower limbs.

\section{Background}

The incidence of distal femoral fractures due to high-energy trauma increased gradually, accounting for $1 \%$ of all fractures and $4-6 \%$ of femoral fractures[1-4]. Currently, patients with distal femoral fractures are primarily treated with early surgical intervention. The most common options include distal femur 
locking plates, compression plates, variable angle locking plates, and less invasive stabilization system (LISS) plates[5-6]. In recent years, continuous improvement of internal fixation devices and surgical techniques has improved the therapeutic effect of femoral fractures. However, malunion of the distal femoral fracture and malalignment of lower extremities still occur[7-8]. Studies have shown that nonunion rates for distal femoral fractures can be as high as 34 percent and present a huge challenge to the orthopaedic surgeon[9-10]. Phillips et al.[11] showed that the incidence of symptoms of mild arthritis of the lower limb knee joint in patients with femoral fracture malunion and malalignment was significantly higher than that in the healthy side, and more obvious symptoms of traumatic arthritis (TA) of the knee joint may occur in long-term follow-up[12]. Nonunion or malunion of fractures can disrupt normal joint movement, leading to non-physiological stress and TA of the knee[13-14].

In order to identify the pathogenesis of traumatic arthritis and the effects of different interventions for traumatic fractures of lower extremities, some scholars have done a lot of researches using techniques such as imaging, biomechanics and finite element analysis[15-17]. Palmu et al.[18] investigated and studied femoral fracture patients over 10 years old, and found 6 cases of residual deformity after fracture healing, which is positively correlated with knee arthritis. It is not infrequent for early knee fractures in patients with previous distal femoral fractures to develop severe arthritis and require total knee arthroplasty. Accordingly, improved biomechanical understanding of tibial varus or valgus malunions will help to formulate improved treatment algorithms.

Therefore, a biomechanical study was conducted using the cadaver models of middle and lower femoral fractures. The fractures were fixed at different varus and valgus deformities, and the stress distribution on the medial and lateral plateau of the femur under weight-bearing load were measured. The study was to investigate the changes of the stress on the medial and lateral plateaus after tibial fracture malunion by comparing with the normal stress, and to identify the relationship between the stress on the plateaus and the residual varus or valgus deformity. The ultimate goal is to hopefully remind the surgeon to reduce the risk of residual varus or valgus deformity during the treatment of femoral fractures.

\section{Methods}

This study has been reviewed and approved by the Institutional Review Board (IRB) of the Third Hospital of Hebei Medical University and that it conformed to the provisions of the Declaration of Helsinki. The experimental flowchart of this biomechanical study was summarized in Fig. 1.

\section{Specimen preparation}

Fourteen formalin-soaked specimens were all adult male donated bodies( Provided by the department of human anatomy, Hebei Medical University). The body height of the specimen was $172 \mathrm{~cm}$ on average (range, from 163 to $182 \mathrm{~cm}$ ). The age when donated was 55 years on average (range, from 40 to 65 years old). Samples from those who had rheumatism, tuberculosis, or tumors were excluded. The specimens were then examined with digital radiography and excluded if they were identified to have osteoporosis, pathological or anatomical deformity, irregular joint surface, or other imaging abnormalities. All samples 
were required to have intact ligaments and tendons around knee joint and unbroken joint capsule. The muscular tissue of each specimen was removed. Then, the upper part of the femur and the distal part of the tibial and fibula were dissected. The remaining femur and tibial and fibula were both about $25 \mathrm{~cm}$ in length. The specimens were maintained in standby packages to prevent dehydration, and kept at $-20^{\circ} \mathrm{C}$ for cryopreservation.

\section{Establishment of femoral fracture malunion model}

The cadaver specimens were thawed at room temperature for 12 hours before the experiment. The middle and lower femoral fracture models were created, and fixed using locking plate and screws at various residual valgus deformity (3 degrees, 7 degrees, 10 degrees), neutral position (0 degrees, anatomically reduced), or varus deformity (3 degrees, 7 degrees, 10 degrees), respectively. A horizontal incision about $3-4 \mathrm{~cm}$ in length was made at the level of the joint space on each side. The subcutaneous fat was separated, the bursa was cut, and the joint space was exposed. The anterior and posterior cruciate ligaments and the medial and lateral menisci were preserved so as not to affect the normal distribution of the contact stress of knee joint.

\section{Specimen assembled to biomechanical testing machine}

The specimen was firstly erected, and the femur end was fixed perpendicularly to the homemade clamp with the use of denture base resin and solution (Type II self-setting dental powder and tray water). After the dental powder and tray water solidified, the tibial end of the specimen was fixed in the same way. Then the two-ends of the clamp were assembled to the biomechanical testing machine (Electroforce 3520-AT, Bose company, USA), and the fixed position of the femoral and tibia stumps was adjusted to make the lower limb mechanical axis close to the position when standing naturally (Fig. 2).

\section{Stress measurement and data collection}

An ultra-low pressure sensitive film ( 0.5 to $2.5 \mathrm{MPa}$ ) was used to measure the contact pressure on the medial and lateral plateaus of the femur. The pressure sensitive film is trimmed into a suitable shape and sealed in a polyethylene film bag. The total thickness of the pressure sensitive film and the polyethylene film bag is controlled to $250 \mu \mathrm{m}$. The fixed specimen was loaded with a tension of $200 \mathrm{~N}$ to pull the knee joint, and the pressure sensitive films were carefully placed under the knee meniscus separately through the medial and lateral incisions (Fig. 3). The incisions were then sutured tightly to close the joint capsule. After stabilization, the specimen was pressurized to $200 \mathrm{~N}$ at $10 \mathrm{~N} / \mathrm{s}$ to eliminate creep. A vertical load was applied on the specimen at a rate of $10 \mathrm{~N} / \mathrm{s}$ to $400 \mathrm{~N}$ for $2 \mathrm{~min}$. Then, the pressure sensitive film was carefully removed from the knee joint. As the pressure-sensitive film material varies with the humidity and temperature, the color development is also different. Therefore, humidifiers and air conditioners are used in the experiment to keep the indoor temperature at $25 \sim 30^{\circ} \mathrm{C}$ and the relative humidity at $35 \% \mathrm{RH} \sim 80 \%$ $\mathrm{RH}$. 
The stress values of the ultra-low pressure sensitive films were read with the use of FPD-305E densitometer and FPD-306E pressure transducer (Fuji Company, Japan). The contact area of each pressure sensitive film was divided into four quadrants (front outer, front inner, rear inner and rear outer). Five points were selected in each quadrant for stress value reading. A total of 20 values in each film were recorded, and the average was calculated for final analysis.

\section{Statistical analysis}

SPSS 21.0 software (SPSS, Chicago, IL, USA) was applied for statistical analysis. The variables in this study include stress values and angles of varus or valgus deformity. The normality of the stress data is verified using the Shapiro-Wilk test, and the variance consistency is verified using the Levene test. Normally distributed measurement data was recorded as mean \pm standard deviation. The stress values on the medial and lateral plateau of the femur at different positions were calculated and recorded, respectively. The analysis of variance (ANOVA) for random block groups was used to compare the stress values on the medial or lateral plateau at different angles of femur varus or valgus deformity under vertical load. The Student-Newman-Keuls (SNK) test was applied to make pairwise comparisons between the multiple sample measurements. Differences in the stress data between medial and lateral plateaus were tested using the paired samples $t$ test. A $P$-value less than 0.05 indicated a statistically significant difference.

\section{Results}

\section{The stress distribution on the medial plateau of femur measured at different varus and valgus deformities}

The stress values on the medial plateau of femur measured at different varus and valgus deformity under $400 \mathrm{~N}$ vertical load are summarized in Table 1. ANOVA for random block groups revealed statistically significant differences in the stress values on the medial plateau among different varus and valgus deformities (including the neutral position) $(F=85.869, P<0.001)$. SNK test revealed statistically significant differences in the stress values on the medial plateau between pairwise comparisons among 10 degrees, 7 degrees and 3 degrees varus deformities, neutral position ( 0 degree) and 3 degrees, 7 degrees, 10 degrees valgus deformities. The stress values on the medial plateau measured at 3 degrees, 7 degrees and 10 degrees varus deformities were significantly higher than that measured at neutral position (all $P<0.05$ ). On the contrary, the stress values on the medial plateau measured at 3 degrees, 7 degrees and 10 degrees valgus deformities were significantly lower than that measured at neutral position (all $P<0.05$ ). From 10 degrees of valgus deformity to 10 degrees of varus deformity, the stress values on the medial plateau of the femur increased proportionably (Fig. 4, Fig. 5). 
Table 1

The stress values (Mpa) on the medial and lateral plateau of femur measured on the models of different varus and valgus deformity under $400 \mathrm{~N}$ vertical load

\begin{tabular}{|c|c|c|c|c|}
\hline Deformity Angle & $\begin{array}{l}\text { Medial plateau } \\
(n=14)\end{array}$ & $\begin{array}{l}\text { Lateral plateau } \\
(n=14)\end{array}$ & t-value & P-value \\
\hline Valgus 10 degrees & $0.743 \pm 0.092$ & $1.567 \pm 0.158$ & 16.79 & $<0.001^{c}$ \\
\hline Valgus 7 degrees & $0.890 \pm 0.137$ & $1.367 \pm 0.101$ & 10.42 & $<0.001^{c}$ \\
\hline Valgus 3 degrees & $1.007 \pm 0.127$ & $1.244 \pm 0.136$ & 4.767 & $<0.001^{c}$ \\
\hline $\begin{array}{l}\text { Neutral position } \\
(0 \text { degree })\end{array}$ & $1.162 \pm 0.114$ & $1.103 \pm 0.144$ & 1.741 & $0.094^{c}$ \\
\hline Varus 3 degrees & $1.277 \pm 0.121$ & $1.059 \pm 0.239$ & -3.048 & $0.005^{c}$ \\
\hline Varus 7 degrees & $1.447 \pm 0.145$ & $0.898 \pm 0.164$ & -9.374 & $<0.001^{\mathrm{c}}$ \\
\hline Varus 10 degrees & $1.652 \pm 0.153$ & $0.809 \pm 0.118$ & -16.31 & $<0.001^{c}$ \\
\hline F-value & 85.869 & 39.730 & & \\
\hline P-value & $<0.001^{a}$ & $<0.001^{b}$ & & \\
\hline \multicolumn{5}{|c|}{$\begin{array}{l}\text { Note: a: Comparison of differences in the stress data on the medial plateau of femur at different } \\
\text { deformed angle. b: Comparison of differences in the stress data on the lateral plateau of femur at } \\
\text { different deformed angle. c: Comparison of differences in the stress data between medial and lateral } \\
\text { plateau of femur at different deformed angles. }\end{array}$} \\
\hline
\end{tabular}

The sress distribution on the lateral plateau of femur measured at different varus and valgus deformities

The stress values on the lateral plateau of femur measured at different varus and valgus deformity under $400 \mathrm{~N}$ vertical load are recorded and summarized (Table 1). ANOVA for random block groups revealed statistically significant differences in the stress values on the lateral plateau among different varus and valgus deformities (including the neutral position) $(F=39.730, P<0.001)$. There were statistically significant differences between pairwise comparisons among 10 degrees, 7 degrees and 3 degrees varus deformities, neutral position and 3 degrees, 7 degrees, 10 degrees valgus deformities according to SNK test. The stress values on the lateral plateau measured at 3 degrees, 7 degrees and 10 degrees valgus deformities were significantly higher than that measured at neutral position (all $P<0.05$ ). Conversely, the stress values on the lateral plateau measured at 3 degrees, 7 degrees and 10 degrees varus deformities were significantly lower than that measured at neutral position (all $P<0.05$ ). From 10 degrees of valgus deformity to 10 degrees of varus deformity, the stress values on the lateral plateau of the femur decreased proportionably (Fig. 6, Fig. 7).

Comparison of the stress values between medial and lateral plateaus of the femur 
Under $400 \mathrm{~N}$ vertical load, the stress on the medial plateau of the femur was $1.162 \pm 0.114 \mathrm{MPa}$ at the neutral position, and the value of $1.103 \pm 0.144 \mathrm{MPa}$ on the lateral plateau of the femur. However, there was no statistical difference between the medial plateau and the lateral plateau in the neutral position $(\mathrm{t}=$ $1.741, P=0.094)$. Under $400 \mathrm{~N}$ vertical load, the stress values on the medial plateau of femur were significantly higher than the corresponding values on the lateral plateau at 3 degrees, 7 degrees, 10 degrees varus deformity positions in addition to neutral position, respectively (all $P<0.05$ ), and significantly lower than the corresponding values on the lateral plateau at 3 degrees, 7 degrees, 10 degrees valgus deformity positions, respectively (all $P<0.05$ ).

\section{Discussion}

Femoral fractures are common injuries in traumatic orthopedics. Open or closed reduction and internal fixation is the standard treatment options for displaced femoral fractures. However, during the closed reduction and intramedullary nailing, the fractures can not be always reduced anatomically, and various residual fracture deformity and complications may occur. Winquist et al.[19] reported that 520 cases of femoral fractures were treated with intramedullary nail, and $8 \%$ of the patients had external rotation deformity of more than 10 degrees. Braten et al.[20] measured the anteversion angle of bilateral femur in 110 patients with interlocking intramedullary nails for unilateral femoral fractures, and 21 (19\%) of them had rotation deformity. The change in the mechanical axis of the lower limbs after fracture malunion can lead to the changes in the tension of the ligaments and joint capsules, and patella malacia in a long time. If not corrected in time, it can affect the biomechanical characteristics of knee and ankle joints and ultimately result in TA, following impaired knee function. Brouwer et al.[21] analyzed the data of 1501 patients (2664 lateral knees), and found that there was a significant correlation between malalignment of lower extremities and the occurrence and progression of arthritis, especially in overweight patients. Maquet[22] also believed that the uneven distribution of stress on the joint surface and the excessive stress concentration is one of the important causes of traumatic arthritis of the knee. Therefore, malunion and malalignment of the lower limb is an important contributing factor to traumatic arthritis of knee joint.

Alignment of the lower limb is a prerequisite for ensuring a reasonable distribution of joint stress. Under normal conditions, the lower limb mechanical axis is from the center of the hip joint to the center of the ankle joint, passing through the center of the knee joint, so that the load and stress on the knee joint can be reasonably dispersed[23]. When malunion and malalignment occurs after femoral fracture, the contact characteristics of knee joint will be altered. In patients with knee varus deformity, the mechanical axis of the lower limbs inclines from the center of the knee to the medial plateau, which causes the stress distribution within the joint to be redistributed, so that the load is mainly concentrated on the medial plateau of the knee. Our findings reveal that the stress on the medial plateau of femur is significantly higher at varus deformities when compared with those measured at the neutral position, and significantly higher than the corresponding stress values on the lateral plateau. When the knee is at valgus position, the mechanical axis is offset laterally and the lateral compartment load increases. We also find that the stress on the lateral plateau is significantly higher at valgus deformities than the stress on the lateral plateau measured at neutral position as well as the corresponding data measured on the medial plateau. 
Studies have shown that the high stress load on joints can increase the damage pressure of articular cartilage, accelerate the wear of articular cartilage, and is an important factor leading to cartilage degradation[24]. Therefore, in order to reduce the risk of long-term arthritis in patients, orthopedic surgeons should reduce the fractures anatomically to avoid the malalignment and malunion of lower limbs fractures[25].

In biomechanics research, the contact position, contact area and contact stress between joint surfaces are often measured. At present, there are a variety of experimental measurement methods for joints, including 3D photoelastic measurement, pressure sensing and finite element analysis[26-27], but these methods have a large error in the measurement of joint stress and the operation method is relatively complex. Therefore, we choose pressure-sensitive film technology, which can not only directly measure the pressure value, but also can measure the contact area, intuitively see the distribution of pressure, has obvious advantages.

This study has some limitations. Firstly, the sample size is small due to the limited source of specimens, which will reduce the credibility of experimental data (type II error). Secondly, this study is based on cadaver specimens and can not take the action of muscles into consideration. Thirdly, dynamic load on the knee joint was not taken and the current model can not simulate the stress distribution during knee motion. However, the current findings guarantee further biomechanical studies on the stress distribution of knee joint under dynamic load, clinical studies on the quantitative relationship between residual malunion and traumatic arthritis, and the endeavor to reduce complex fractures anatomically in closed fashion or minimally invasive way.

\section{Conclusions}

In conclusion, femoral fracture malunion with residual varus or valgus deformity can lead to significant changes in the stress distribution and contact characteristics of the knee joint. Therefore, orthopedic surgeons should reduce the fractures anatomically to avoid malunion of the fractures and malalignment of the affected lower extremities, accordingly improving the functional recovery and reducing the risk of traumatic arthritis in the long term.

\section{Abbreviations}

LISS

Less invasive stabilization system; TA:Traumatic arthritis ANOVA

Analysis of variance; SNK:Student-Newman-Keuls

\section{Declarations}

\section{Ethics approval and consent to participate}


This study has been reviewed and approved by the Institutional Review Board (IRB) of the Third Hospital of Hebei Medical University and that it conformed to the provisions of the Declaration of Helsinki.

\section{Consent for publication}

Manuscript does not contains any individual person's data.

\section{Availability of data and materials}

The datasets during and/or analyzed during the current study are available from the corresponding author on reasonable request.

\section{Competing interest}

The authors declare that they have no competing interests.

\section{Funding}

This study was supported by the National Natural Science Foundation of China (Grant No. 81401789) and Hebei National Science Foundation-Outstanding Youth Foundation (Grand No. H2017206104). The funds above completely financed the costs of the study and contributed to the design of the study,interpretation of data, and revising the manuscript.

\section{Authors' contributions}

WC and PH designed the study and searched relevant studies. DZ,WL C, YB Z, TZ and NW analyzed and interpreted the data. ML and LJ M wrote the manuscript and contributed equally to this work. WC contributed most in the revision of this manuscript and approved the final version of the manuscript. All authors read and approved the final manuscript.

\section{Acknowledgments}

The authors are grateful to the Institute of Orthopedic Reasearch of Hebei province for their excellent technical support.

\section{References}

1. Court-Brown CM, Caesar B. Epidemiology of adult fractures: a review. Injury. 2006;37:691-7.

2. Kolmert L, Wulff K. Epidemiology and treatment of distal femoral fractures in adults. Acta Orthop Scand. 1982;53:957-62.

3. Phelps EE, Tutton E, Griffin X, Baird J. A qualitative study of patients' experience of recovery after a distal femoral fracture. Injury. 2019;50:1750-5.

4. $\mathrm{Ma} \mathrm{CH}$, Chiu $\mathrm{YC}, \mathrm{Wu} \mathrm{CH}$, et al. Plate-on-plate technique for treating peri-implant fractures of distal femoral locking plate: a retrospective study of 11 patients. Arch Orthop Trauma Surg. 
2019;139:1245-51.

5. Tank JC, Schneider PS, Davis E, et al. Early mechanical failures of the synthes variable angle locking distal femur plate. J Orthop Trauma. 2016;30:e7-11.

6. McDonald TC, Lambert JJ, Hulick RM, et al. Treatment of distal femur fractures with the DePuySynthes variable angle locking compression plate. J Orthop Trauma. 2019;33:432-7.

7. Krettek C, Miclau T, Blauth M, Lindsey RW, Donow C, Tscherne H. Recurrent rotational deformity of the femur after static locking of intramedullary nails: case reports. J Bone Joint Surg Br. 1997;79:48.

8. Goodier WD, Calder PR. External fixation for the correction of adult post-traumatic deformities. Injury. 2019;50:36-44.

9. Ricci WM, Streubel PN, Morshed S, Collinge CA, Nork SE, Gardner MJ. Risk factors for failure of locked plate fixation of distal femur fractures: an analysis of 335 cases. J Orthop Trauma. 2014;28:83-9.

10. Gwathmey FW Jr, Jones-Quaidoo SM, Kahler D, Hurwitz S, Cui Q. Distal femoral fractures: current concepts. J Am Acad Orthop Surg. 2010;18:597-607.

11. Phillips JR, Trezies AJ, Davis TR. Long-term follow-up of femoral shaft fracture: Relevance of malunion and malalignment for the development of knee arthritis. Injury. 2011;42:156-61.

12. Wang JW, Chen WS, Lin PC, Hsu CS, Wang CJ. Total knee replacement with intra-articular resection of bone after malunion of a femoral fracture: can sagittal angulation be corrected? J Bone Joint Surg Br. 2010;92:1392-6.

13. Svoboda SJ, McHale CK, Belkoff SM, Cohen KS, Klemme WR. The Effects of Tibial Malrotation on the Biomechanics of the Tibiotalar Joint. Foot Ankle Int. 2002;23:102-6.

14. Wang XS, Zhou YX, Shao HY, Yang DJ, Huang Y, Duan FF. Total Knee Arthroplasty in Patients with Prior Femoral and Tibial Fractures: Outcomes and Risk Factors for Surgical Site Complications and Reoperations. Orthop Surg. 2020;12:210-7.

15. Sarai T, Inoue T, Fujiwara K, Kuramoto K. Dynamic finite element analysis of impulsive stress waves propagating from distal end of femur. Acta Med Okayama. 2012;66:409-15.

16. McErlain DD, Milner JS, Ivanov TG, Jencikova-Celerin L, Pollmann SI, Holdsworth DW. Subchondral cysts create increased intra-osseous stress in early knee OA: A finite element analysis using simulated lesions. Bone. 2011;48:639-46.

17. Tsui CP, Tang CY, Leung CP, et al. Active finite element analysis of skeletal muscle-tendon complex during isometric, shortening and lengthening contraction. Biomed Mater Eng. 2004;14:271-9.

18. Palmu SA, Lohman M, Paukku RT, Peltonen JI, Nietosvaara Y. Childhood femoral fracture can lead to premature knee-joint arthritis. 21-year follow-up results: a retrospective study. Acta Orthop. 2013;84:71-5.

19. Winquist RA, Hansen ST Jr, Clawson DK. Closed intramedullary nailing of femoral fractures. A report of five hundred and twenty cases. J Bone Joint Surg Am. 1984;66:529-39. 
20. Braten M, Terjesen T, Rossvoll I. Torsional deformity after intramedullary nailing of femoral shaft fractures. Measurement of anteversion angles in 110 patients. J Bone Joint Surg Br. 1993;75:799803.

21. Brouwer GM, van Tol AW, Bergink AP, et al. Association between valgus and varus alignment and the development and progression of radiographic osteoarthritis of the knee. Arthritis Rheum. 2007;56:1204-11.

22. Maquet PGJ. Biomechanics of the knee. With application to the pathogenesis and the surgical treatment of osteoarthritis. Brelin Springer- Verlag. 1984: 165.

23. Zhu Z, Wang L, Li D, et al. Motion analysis on patients with knee osteoarthritis merged with varus deformity. J Biomed Eng. 2018;35:38-44.

24. Hamerman D. The biology of osteoarthritis. N Engl J Med. 1989;18:1322-30.

25. Sanders R, Anglen JO, Mark JB. Oblique osteotomy for the correction of tibial malunion. J Bone Joint Surg Am. 1995;77:240-6.

26. Giri D, Jouaneh M, Stucker B. Error sources in a 3-D reverse engineering process. Precis Eng. 2004;28:242-51.

27. Li L, Yang L, Zhang K, Zhu L, Wang X, Jiang Q. Three-dimensional finite-element analysis of aggravating medial meniscus tears on knee osteoarthritis. J Orthop Translat. 2019;20:47-55.

\section{Figures}


Specimens with rheumatism, tuberculosis, tumors or imaging abnormalities were excluded

The middle and lower femoral fracture models were created, and fixed using locking plate and screws residual valgus deformity $\left(3^{\circ}, 7^{\circ}, 10^{\circ}\right)$ or varus deformity $\left(3^{\circ}, 7^{\circ}, 10^{\circ}\right)$, respectively, and fixed on the BOSE Electroforce 3250-AT biomechanics machine

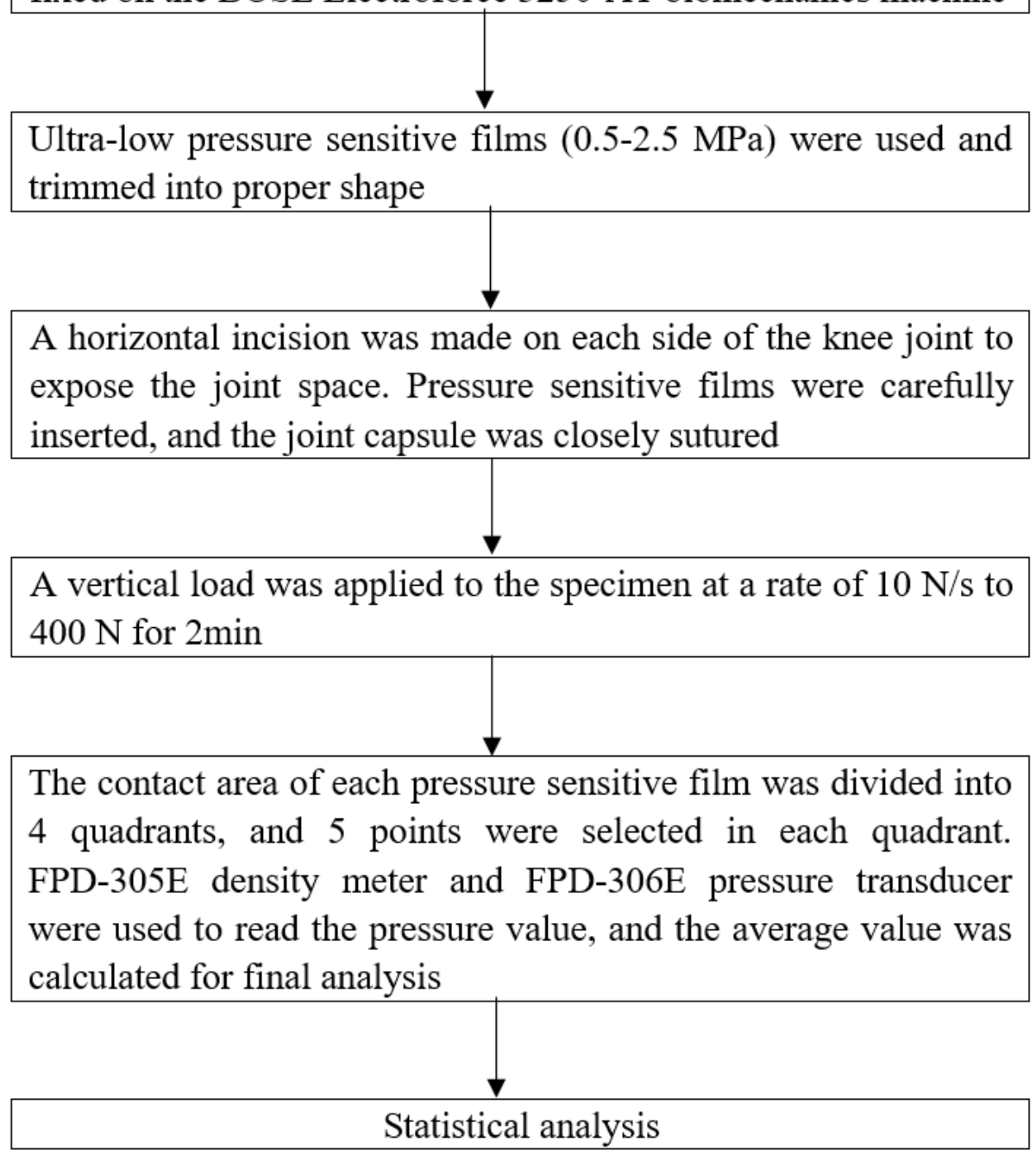

\section{Figure 1}

The experimental flowchart of the biomechanical study on the stress distribution of knee joint after malunion of femoral fracture. 


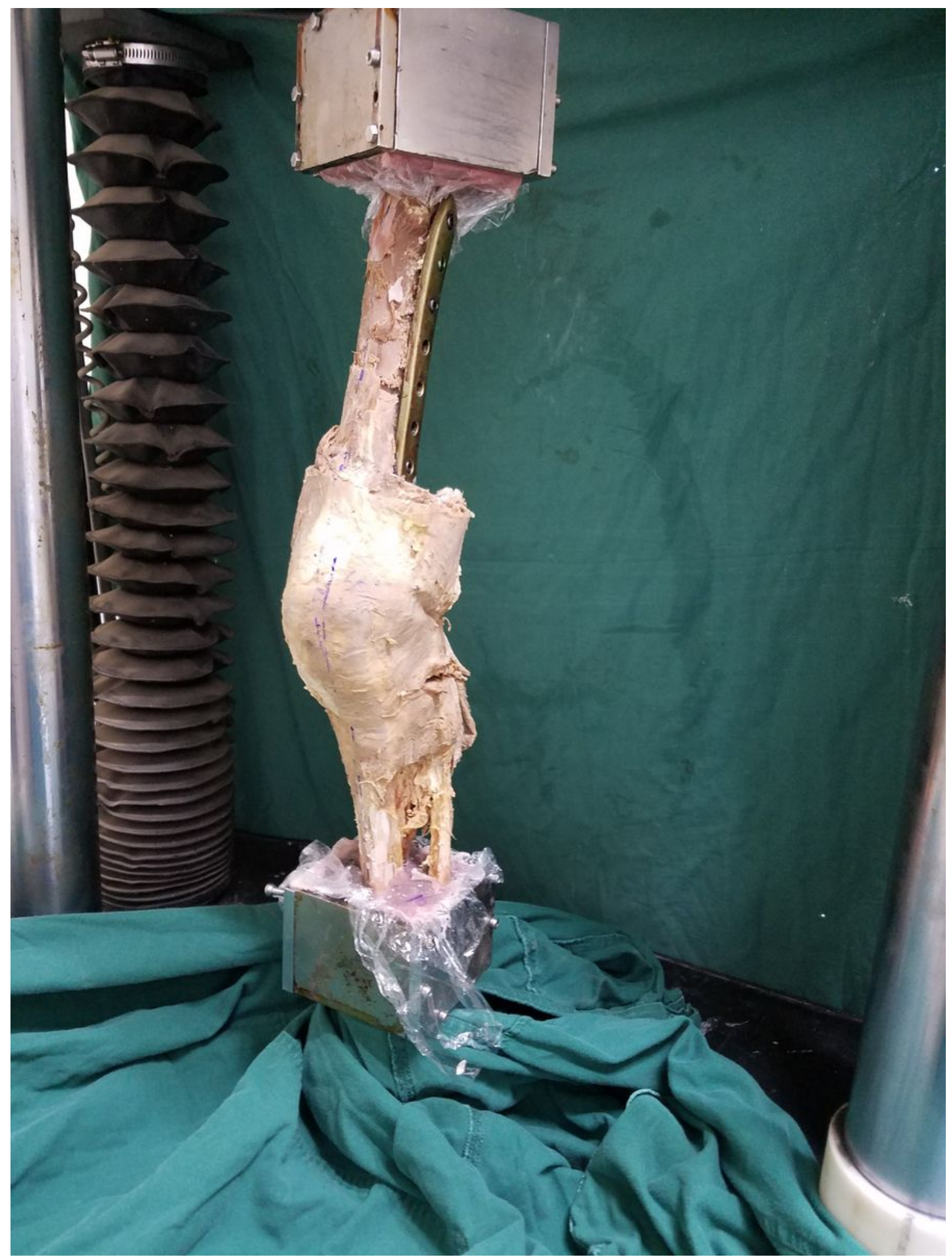

Figure 2

The specimens were assembled to the BOSE Electroforce 3520-AT biomechanical testing machine. 


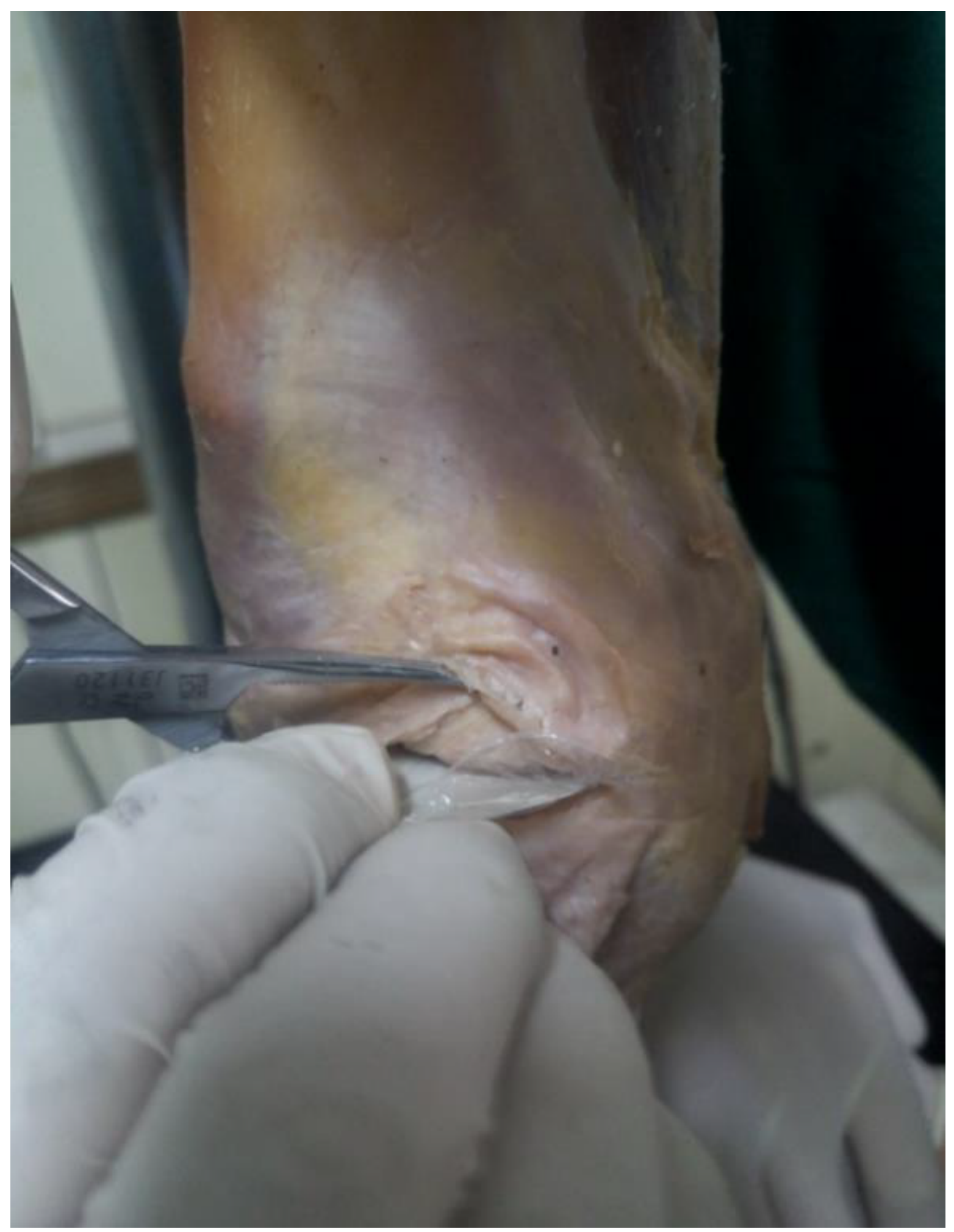

Figure 3

The pressure sensitive films were carefully placed under the meniscus. 

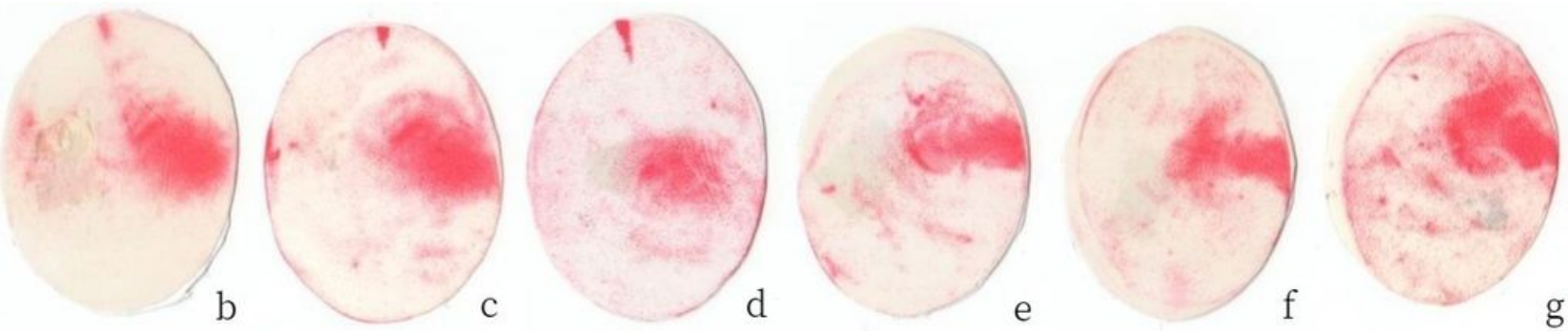

\section{Figure 4}

Color changes of the ultra-low pressure sensitive films on the medial plateau at different angles of varus and valgus deformities of the femur. a, valgus 10 degrees; $b$, valgus 7 degrees; $c$, valgus 3 degrees; $d$, neutral position 0 degree; e, varus 3 degrees; $f$, varus 7 degrees; $g$, varus 10 degrees.

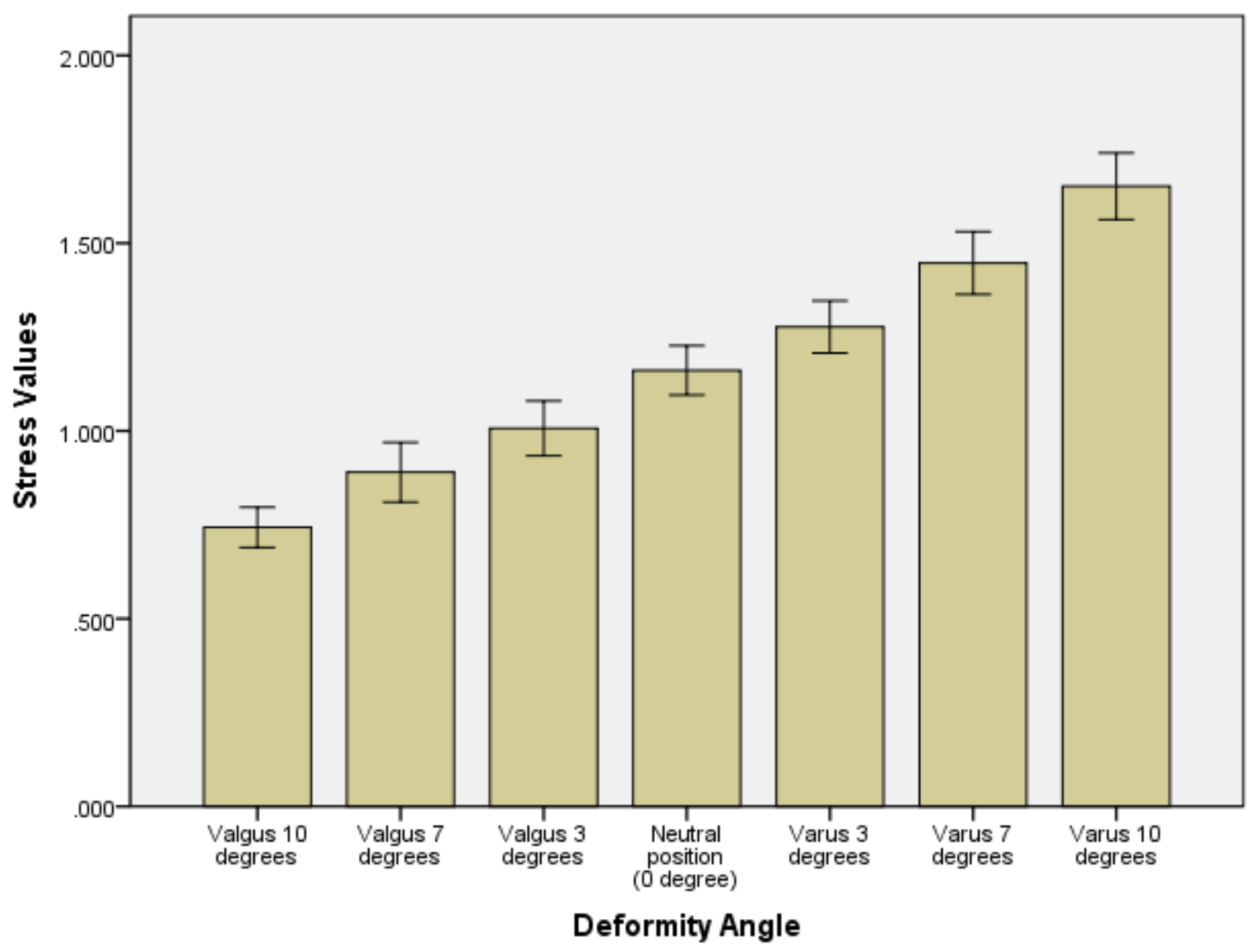

Error bars: $95 \% \mathrm{Cl}$

\section{Figure 5}

From 10 degrees of valgus deformity to 10 degrees of varus deformity, the stress values on the medial plateau of the femur increased proportionably. 

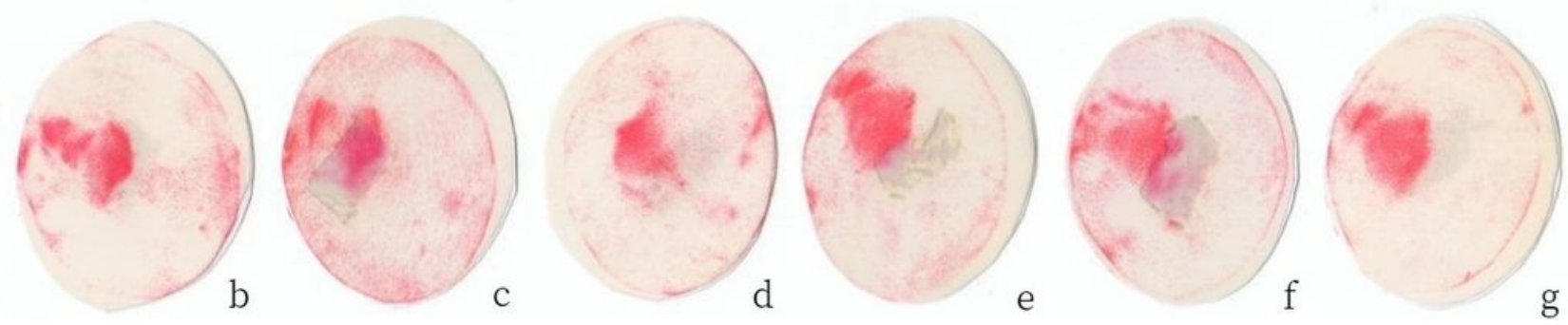

Figure 6

Color changes of the ultra-low pressure sensitive films on the lateral plateau at different angles of varus and valgus deformities of the femur. a, valgus 10 degrees; $b$, valgus 7 degrees; $c$, valgus 3 degrees; $d$, neutral position 0 degree; e, varus 3 degrees; $f$, varus 7 degrees; $g$, varus 10 degrees.

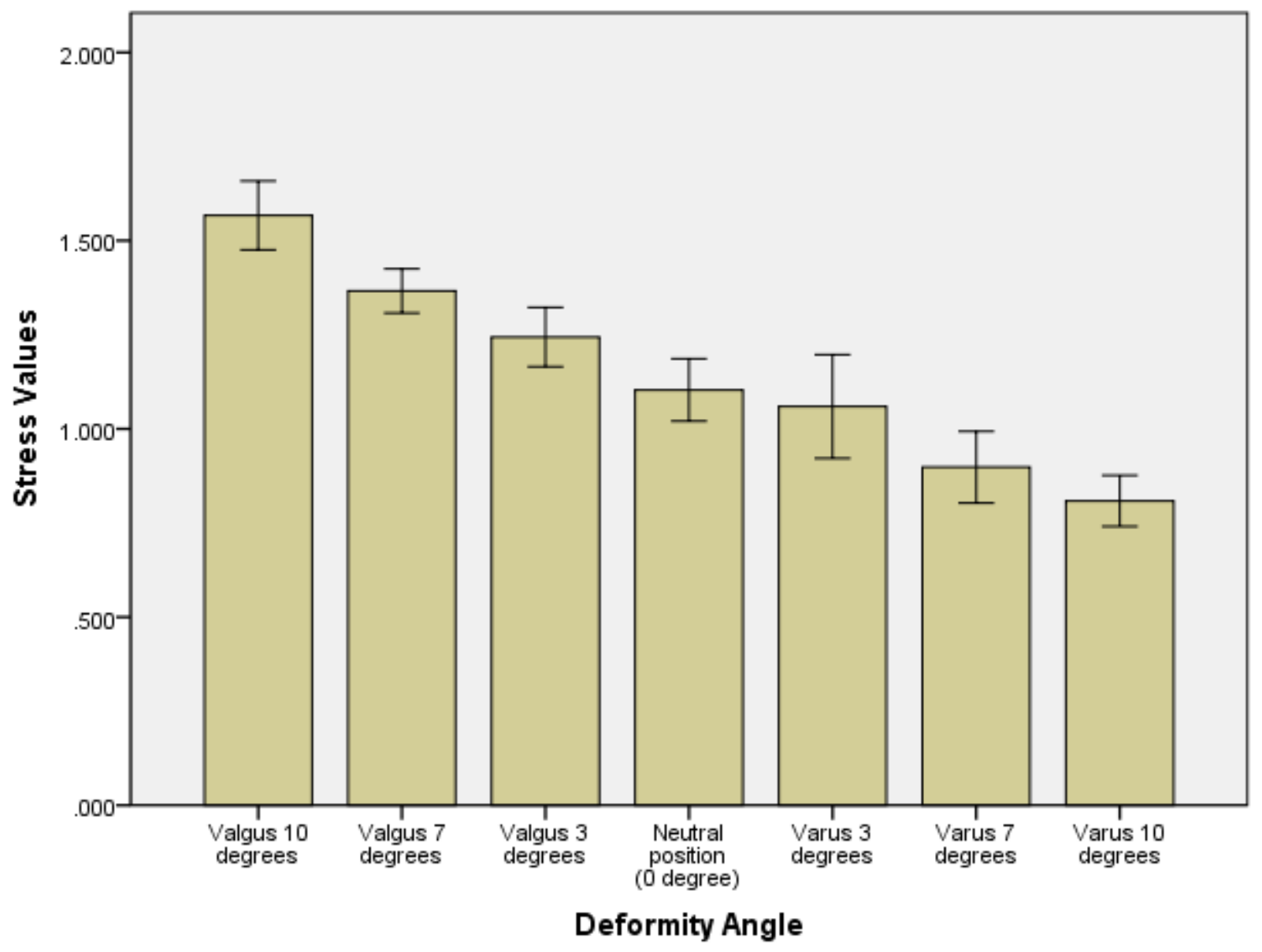

Error bars: $95 \% \mathrm{Cl}$

\section{Figure 7}

From 10 degrees of valgus deformity to 10 degrees of varus deformity, the stress values on the lateral plateau of the femur decreased proportionably. 